\title{
Liquid-gas transition of neon in quasi-one-dimensional environments
}

\author{
L. Brualla \\ International School for Advanced Studies, SISSA, and INFM DEMOCRITOS National Simulation Center, \\ Via Beirut I-34014 Trieste, Italy \\ M. C. Gordillo \\ Departamento de Ciencias Ambientales, Facultad de Ciencias Experimentales, Universidad Pablo de Olavide, Carretera de Utrera km 1, \\ 41013 Sevilla, Spain
}

(Received 27 May 2003; revised manuscript received 10 July 2003; published 29 August 2003)

\begin{abstract}
We characterize the behavior of a system of $\mathrm{Ne}^{20}$ atoms in a pure one-dimensional environment between 12 and $30 \mathrm{~K}$ by means of path integral Monte Carlo calculations. This is a reasonable model to describe neon absorbed inside a narrow carbon nanotube. When embedded in a bundle of those tubes, this quasi-onedimensional system can undergo a liquid-gas phase transition if nanotubes are narrow and close enough.
\end{abstract}

DOI: $10.1103 /$ PhysRevB.68.075423

PACS number(s): 68.90. $+\mathrm{g}$, 05.30. $-\mathrm{d}$

\section{INTRODUCTION}

One-dimensional (1D) systems were little more than a theoretical curiosity until the discovery of carbon nanotubes (CN's) in 1991. ${ }^{1}$ These are long and narrow cylinders whose walls are made of graphite sheets and, in their native form, are closed by caps. However, it is possible to open them by chemical or other means, allowing the absorption of species of the right size inside. There exist nanotubes of many different radii $(R)$, even though the most abundant ones have $R=6.8 \AA[(10,10)$ in the standard nomenclature $]$. The narrowest tubes obtained by ordinary means are the $(5,5)$ ones, with a radius of $3.4 \AA$, but $(4,4)$ cylinders can be synthesized inside a template, such as a zeolite. ${ }^{2}$ When absorbed inside a $(5,5)$ tube, small atoms or molecules could be described reasonably well as a one-dimensional system, specially if they fit tightly, as in the case of $\mathrm{H}_{2} \cdot{ }^{3}$ Unfortunately, in other cases (e.g., $\mathrm{He}^{4}$ ) that description is just an approximation. ${ }^{4,5} \mathrm{An}$ even narrower environment is achieved using the interchannels in between every three tubes of a bundle of nanotubes: in a $(10,10)$ tube those channels would permit the absorption of species of around $3 \AA$ of radius. ${ }^{6}$ However, the experimental reports of having observed atoms or molecules in that particular location are scarce. ${ }^{7}$ In fact, there is even a work that states that neon is not absorbed in the interstices of a bundle of $(10,10)$ CN's. ${ }^{8}$

In this work we present path-integral Monte Carlo (PIMC) calculations of pure one-dimensional $\mathrm{Ne}^{20}$ at finite temperature, and some zero-temperature calculations on the same system carried out by means of a diffusion Monte Carlo (DMC) technique. To our knowledge, this is the first time that a full many-body calculation is reported for a quantum 1D gas at finite temperature, in contraposition to the several zero-temperature equations of state already reported for several gases ( $\mathrm{He}^{4},{ }^{4,9-12} \mathrm{H}_{2}$, Ref. 3) even though the case of $\mathrm{Ne}$ has not been considered yet. There have been also many studies about what would happen for infinite diluted gases adsorbed inside nanotubes or the interchannels mentioned above (Ref. 13,14, and references therein). Both PIMC an d DMC are standard algorithms, and details about them could be found in Ref. 15 and Refs. 16,17, respectively.
The PIMC method allows the calculation of quantum nondynamical properties of finite-temperature systems by mapping the quantum system onto an equivalent classical model in which each atom is described by a certain number of beads connected by springs. The beads interact with each other by a fraction of the real interatomic potential, that in the Ne case is taken to be the Aziz HFD-B Ne-Ne pair potential. ${ }^{18}$

The PIMC simulations presented in this work were performed at $12,15,20,25$, and $30 \mathrm{~K}$, using a linear density $(\rho)$ range between 0 and $0.34 \AA^{-1}$. Exchanges were not allowed, which implies that $\mathrm{Ne}^{20}$ atoms are not considered bosons, but boltzmanons. The temperature range at which the simulations are done guarantees that this is a good approximation. ${ }^{19,20}$ From PIMC simulations, we obtained the pressure by means of the virial estimator,

$$
p=\frac{N T}{L}-\frac{1}{L} \sum_{i<j} r_{i j} \frac{d V\left(r_{i j}\right)}{d r_{i j}},
$$

where $T$ is the absolute temperature and $L$ is the length of the simulation cell. $V\left(r_{i j}\right)$ is the $\mathrm{Ne}-\mathrm{Ne}$ interatomic potential between two atoms located at a distance $r_{i j}$ from each other. In all cases the number of atoms, $N$, was 30 , and we varied the densities by varying $L$.

On the other hand, DMC is a technique that allows us to obtain the ground state of a particular system by solving its Schrödinger equation by stochastic means. To do so, we need to introduce a trial wave function that summarizes all the information known a priori about the system. The trial function used in the 1D Ne calculation was a Jastrow one with a McMillan correlation factor. The parameter in that correlation factor was previously determined by means of a variational Monte Carlo optimization.

\section{ONE-DIMENSIONAL SYSTEM}

The primary output of a DMC simulation is the energy and, since we are in the ground state, we can get the pressure from 


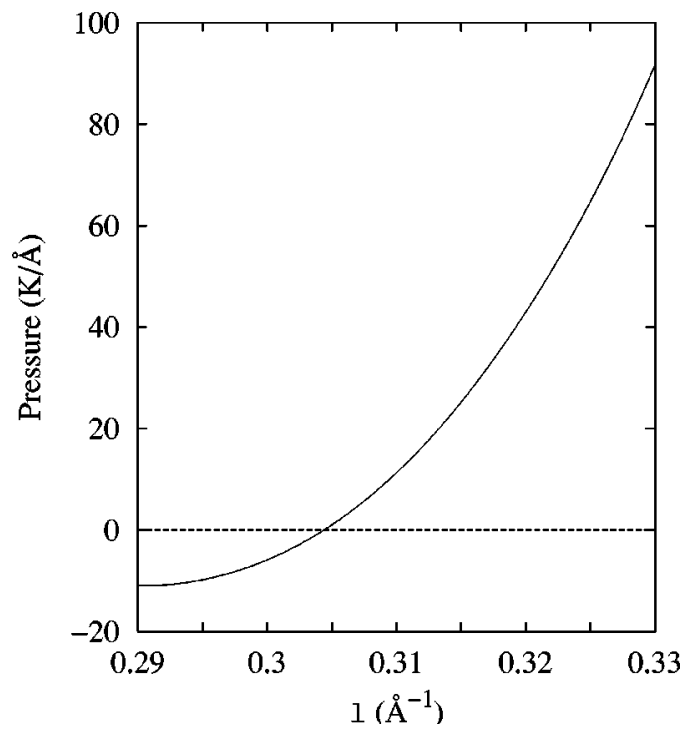

FIG. 1. Pressure as a function of density. Ground state of a pure 1D system of $\mathrm{Ne}$ atoms.

$$
p=\rho^{2} \frac{\partial E}{\partial \rho},
$$

where $\rho$, as before, means 1D density.

Figure 1 is a plot of the pressure of a pure onedimensional system of $\mathrm{Ne}$ atoms as a function of $\rho$. It has been obtained from the ground-state energy by means of Eq. (2). A couple of things are immediately apparent: there is a minimum in the energy that corresponds to the density at which $p=0 \quad\left(\rho_{0}=0.3044 \pm 0.0001 \AA^{-1}\right.$, from a third-grade polynomial fit), and at that density the system is a liquid, since for lower $\rho$ 's the pressure is negative (we have a van der Waals loop). Moreover, the system is bound for $\rho>\rho_{0}$ with a binding energy per particle $E_{0}=-26.7 \pm 0.3 \mathrm{~K}$ at $\rho_{0}$. Neon behavior is similar to that of $1 \mathrm{D} \mathrm{H}_{2},{ }^{3}$ also a liquid in its ground state. The spinodal point for $1 \mathrm{D}$ neon obtained from the calculations is $\rho_{s}=0.2904 \pm 0.0001 \AA^{-1}$.

Since at zero temperature the ground-state phase is liquid, and at infinite temperature all systems must be gases, it seems that there should be a finite temperature at which one would find a liquid-gas phase transition. However, Van Hove $^{21}$ showed in the 1950 s that a one-dimensional phase transition is impossible when we have a finite-ranged interaction, and other authors, ${ }^{22-24}$ proved that a long-range potential decaying faster than $1 / r^{2}$ could not present a phase transition in purely 1D systems. All that means that the liquid-gas critical point seems to be at $0 \mathrm{~K}$, as in the orderdisorder change of a one-dimensional Ising magnet. Unfortunately, the particular characteristics of these 1D arrays make thermodynamical properties at low temperatures difficult to obtain. The reason is the big fluctuations of those properties that appear in any system when it is close to a critical point. Since in 1D those fluctuations are particularly large, we could find that the correlation length at a given density and temperature is larger than the largest simulation cell we can afford. In such a case, we could be trapped in a part of the system that is, for instance, liquidlike, and hence
TABLE I. PIMC energies and pressures for several densities at 12 and $15 \mathrm{~K}$. All these systems have been done twice, using 120 and 30 atoms in order to measure finite-size effects.

\begin{tabular}{lcccc}
\hline \hline$T[\mathrm{~K}]$ & $N$ & $\rho\left[\AA^{-3}\right]$ & $E[\mathrm{~K}]$ & $p[\mathrm{~K} / \AA]$ \\
\hline 15 & 120 & 0.220 & $-13.0(3)$ & $7.8(7)$ \\
& 30 & 0.220 & $-13.2(4)$ & $7.2(6)$ \\
& 120 & 0.100 & $-5.2(2)$ & $2.9(3)$ \\
& 30 & 0.100 & $-5.1(3)$ & $2.9(1)$ \\
12 & 120 & 0.200 & $-14.6(5)$ & $2.8(7)$ \\
& 30 & 0.200 & $-14.6(7)$ & $2.6(2)$ \\
& 120 & 0.100 & $-8.1(4)$ & $1.5(3)$ \\
& 30 & 0.100 & $-8.8(3)$ & $1.44(6)$ \\
\hline \hline
\end{tabular}

obtaining wrong thermodynamic averages from the calculation. To avoid that, we simulated the corresponding 1D neon systems at temperatures at which we were certain that the correlation length was smaller than the simulation cells used. As a check, we performed an additional set of simulations at densities $0.100 \AA^{-1}$ and $0.200 \AA^{-1}$ for $12 \mathrm{~K}$, and $0.100 \AA^{-1}$ and $0.220 \AA^{-1}$ for $15 \mathrm{~K}$, with the same parameters, but with 120 atoms, instead of 30 as we have employed in all other temperatures and densities. The results are given in Table I for the total energy and the pressure. It can be seen that all data are compatible with their smaller cell counterparts and that the error bars are similar in all cases. This would imply that the observables we calculated (total energy per particle and pressure) are reliable.

The pressure data at finite temperatures are given in Fig. 2. All the points correspond to one-dimensional pressures, measured in $\mathrm{K} / \AA$. From top to bottom we have the isotherms for $30 \mathrm{~K}, 25 \mathrm{~K}, 20 \mathrm{~K}, 15 \mathrm{~K}$, and $12 \mathrm{~K}$, with their correspond-

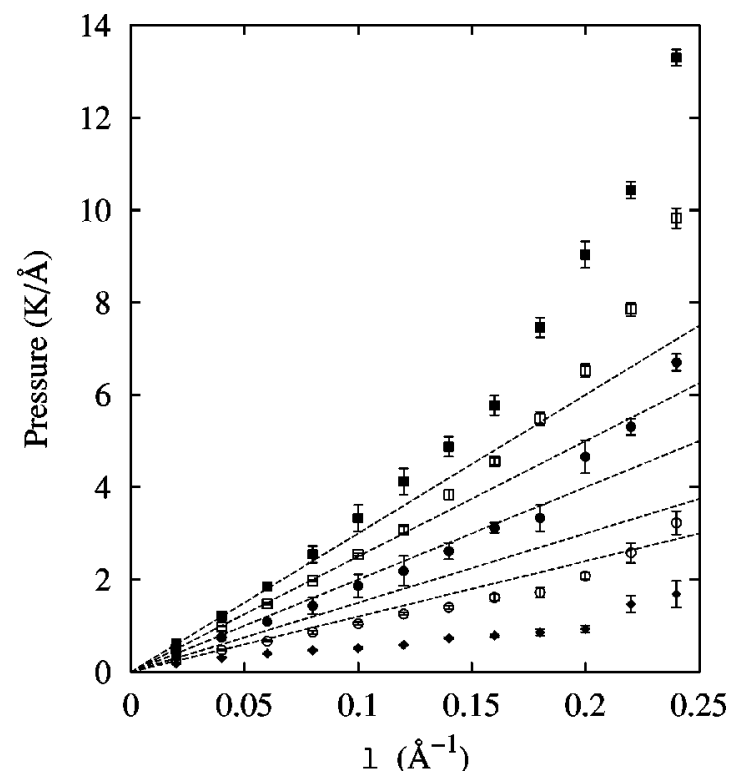

FIG. 2. PIMC pressures of a 1D system of neon atoms along the following isotherms: $30 \mathrm{~K}$ (filled squares), $25 \mathrm{~K}$ (squares), $20 \mathrm{~K}$ filled circles), $15 \mathrm{~K}$ (circles), and $12 \mathrm{~K}$ (filled diamonds). The dashed lines are the ideal gas pressures of a 1D system at the temperatures cited above. 
ing error bars. We displayed also the pressure values of a 1D ideal gas $(p=\rho T)$ at that same temperatures as dashed lines, and in the same order from top to bottom. Obviously, and in line with the previous discussion, in all isotherms the pressure values are monotonically increasing functions of the density, with no van der Waals loops signaling any phase transition. If we analyze the results, there is another evident fact: the temperature has to be raised up to at least $25 \mathrm{~K}$ in order to find a sizable density interval in which the ideal gas approximation is valid $\left(\rho<0.12 \AA^{-1}\right)$. At $20 \mathrm{~K}$ there is a small negative deviation of the ideal behavior at low densities, and a positive deviation at high densities, but the obtained pressures are not very different from the corresponding to an ideal gas. However, the 15 and $12 \mathrm{~K}$ isotherms are far from the ideal gas behavior. On the other hand, at $30 \mathrm{~K}$, the pressure of the gas is greater than the corresponding to the ideal situation. If we describe the departure of the ideal behavior for low densities $\left(\rho<0.12 \AA^{-1}\right)$ by means of a second virial coefficient, we would have a tendency similar to its three-dimensional counterpart: ${ }^{25}$ a negative value at low temperatures, a Boyle temperature of about $25 \mathrm{~K}$, and a positive deviation when we heat up the system further.

In order to translate these pressures into threedimensional ones, the neon atoms have to be supposed adsorbed in a narrow tube $[(5,5)]$ or in the interstitial positions of a $(10,10)$ bundle. Then, we have to divide the 1D pressures by the transversal area of the absorption site. For a $(5,5)$ tube that area is $\pi R^{2}=36.3 \AA^{2}$, while for an interstitial channel it is $52.5 \AA^{2}$.

\section{QUASI-ONE-DIMENSIONAL NEON ABSORBED IN BUNDLES}

Up to now, we have circumscribed ourselves to the case of a single one-dimensional system. However, the carbon nanotubes usually associate themselves forming bundles. In those bundles, every $\mathrm{CN}$ is surrounded by other six tubes forming a triangular lattice in the plane perpendicular to their longitudinal axes. On the other hand, the parallel interchannels in between three of those tubes are disposed in a hexagonal pattern in the same $x y$ plane. The presence of atoms in those adjacent tubes or interchannels exerts a certain influence in the one-dimensional arrays of atoms. In particular, the effect in the pressure is taken into account by adding the term

$$
-\frac{1}{L} \sum_{i j} r_{i j} \frac{d V\left(r_{i j}\right)}{d r_{i j}}
$$

to Eq. (1). Here, $r_{i j}$ is the distance between atoms in different tubes. However, these distances are considerably large, even in the most favorable situations. If the atoms are absorbed inside $(5,5)$ tubes forming a bundle, the minimal distance between them is $\sim 10.2 \AA$. One expects then that the correlation between atoms is minimal, and the pressure effect of the presence of the other tubes in the bundle could be described by a mean-field approximation, ${ }^{11}$

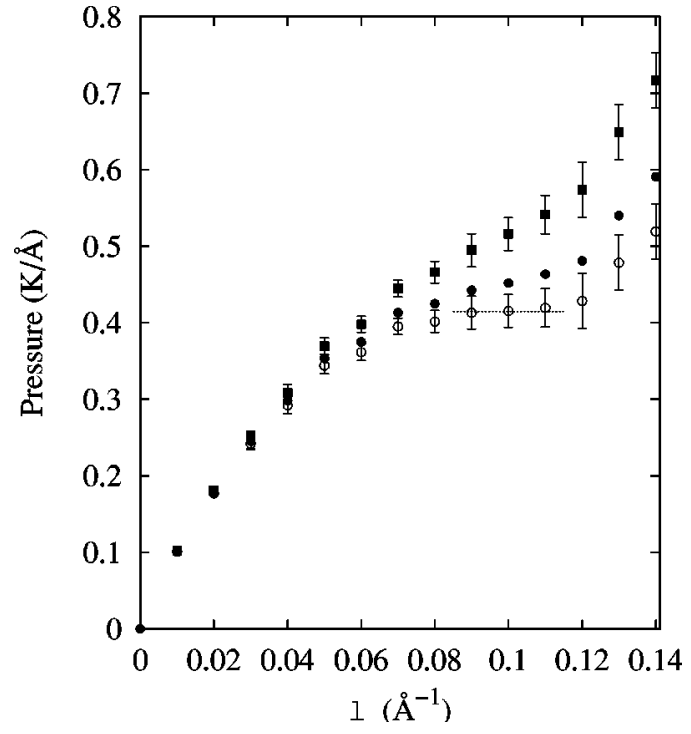

FIG. 3. $12 \mathrm{~K}$ isotherms. Filled squares, pure 1D system. Circles, neon inside $(5,5)$ nanotubes. Filled circles, neon absorbed in the interstices of a $(10,10)$ bundle.

$$
-\rho^{2} \int_{0}^{\infty} r \frac{d V(r)}{d r} d r
$$

The accuracy of this approach has been checked for the energy in the case of $\mathrm{He}^{4}{ }^{26}$

We performed the integral in two cases, $\mathrm{Ne}$ absorbed inside $(5,5)$ tubes forming a bundle, and the same species in the interchannels of a set of $(10,10)$ tubes. In this last configuration, the minimum distance between interchannels is $\sim 9.81 \AA$. For the first case, the integration renders $-10.09 \rho^{2} \mathrm{~K} / \AA$, and in the second, $-6.44 \rho^{2} \mathrm{~K} / \AA$. As in the rest of this work, $\rho$ is the linear density of neon atoms in the corresponding environment, and it is supposed to be the same in all tubes or interchannels. If we want to have the pressure for the three-dimensional systems, we should divide the obtained values, including the corrections, by the accessible perpendicular area, as commented above.

Figure 3 displays the effects of that correction in the $12 \mathrm{~K}$ isotherm. This plot shows the pure 1D system (filled squares), the bundle of $(5,5)$ tubes (circles), and what would happen if the array of atoms were absorbed in the interchannels of the $(10,10)$ bundle (filled circles). Obviously, the error bars are the same in all three cases and are not depicted in the last one for simplicity. The 1D isotherm increases monotonically with density, as it should for a system with no phase transitions. The same happens for interstitial neon, but the case of the $(5,5)$ array of bundles is completely different. It can be seen that there is a density range $0.09 \AA^{-1}<\rho$ $<0.11 \AA^{-1}$ in which the corrected isotherm is flat. This is a signal for a liquid-gas phase transition, since for that critical isotherm there should be at least a point at which $\partial p / \partial v$ and $\partial^{2} p / \partial v^{2}$ are both zero. Thus, the corresponding critical point would be $T_{c} \sim 12 \mathrm{~K}$, with a $\rho_{c}=0.10 \AA^{-1}$. Since there is no phase transition in $1 \mathrm{D}$, the reason for which there is critical temperature is the influence of the surrounding tubes. In fact, this is not the first time that coupling with neighboring tubes 
is the mechanism invoked to induce a phase gas-liquid ${ }^{27,28}$ or a melting ${ }^{29,30}$ transition. There is even an estimation of a gas-liquid transition critical temperature in the case of $\mathrm{Ne}$, using a 3D modified anisotropic Ising model to get $T_{c} \cdot{ }^{31} \mathrm{~A}$ comparison between the number obtained in that approximation $\left(T_{c}=63.8 \mathrm{~K}\right)$ and the one in the present work shows that in the neon case that Ising model is completely inadequate, at least with the parameters given in that reference.

The minimum distance between tubes is not the only variable here, we have also the particular disposition of the 1D arrays in space. ${ }^{28}$ Even though the neon atoms of different interchannels are located closer than if they were absorbed in a $(5,5)$ tube, in the former case, each line of neon atoms is surrounded by three other lines (hexagonal pattern), while in the latter the number of next-neighbor lines is six (triangular pattern). In fact, in a real system of neon in $(10,10)$ interchannels, one should decrease more the temperature to obtain a flat isotherm, as can be deduced from the data of Fig. 3 . This is very difficult in our simulations, due to $1 \mathrm{D}$ correlation problems mentioned above, but it should not be a major problem in an experimental set up. The $(5,5)$ case considered here would be an upper bound for the critical temperature of quasi-1D neon, since $10.2 \AA$ is the smallest possible distance between tubes. For larger tubes, the 1D approximation would be worst.

A word should be said about the possibility of having $\mathrm{Ne}$ inside the interstices of a bundle of $(10,10)$ tubes. Talapatra et $a l .{ }^{8}$ have recently stated that that was impossible. On the other hand, a DMC calculation of the energy for a single atom in such an environment would give a ground-state energy of $-1011.1522 \pm 0.0009 \mathrm{~K}$, i.e., the neon should enter the channels. Two are the reasons for the apparent contradic- tion. The first is the difference between the experimental setup and the scene the calculations imply. Ref. 8 claims that the nanotubes were not prepared in any special way, implying that some of the interchannels would be clogged and unaccessible to the neon atoms. Second, the tubes could be far from the parallel, perfect and regular cylinders assumed in simulations. However, the most important effect would be entropic. In going from a three-dimensional environment to a one-dimensional one, the entropy decreases a fair amount. The effect could be estimated applying the Sakur-Tetrode equation for the entropy of an ideal gas in both cases, and obtaining the difference, that is more or less negative depending on temperature and on the length of the system. However, if the temperature is low enough, the absorption would certainly take place.

\section{CONCLUSIONS}

Summarizing, we found that even though a liquid-gas phase transition is impossible in a pure $1 \mathrm{D}$ environment, the influence of neon atoms absorbed inside neighboring tubes or interchannels could create such a phenomenon. The upper critical temperature possible is that of neon absorbed in the inner part of opened $(5,5)$ tubes forming a bundle, about 12 $\mathrm{K}$. For comparison, the $T_{c}$ of three-dimensional neon is $44.45 \mathrm{~K},{ }^{32}$ and for $\mathrm{Ne}$ on top of a graphite sheet, $15.8 \mathrm{~K}^{33}$

\section{ACKNOWLEDGMENTS}

We thank S. Fantoni, K. E. Schmidt, and J. M. Romero Enrique for fruitful discussions. L.B. acknowledges Italian MIUR-National Research for financial support under Project No. 2001/025/498.
${ }^{1}$ S. Iijima, Nature (London) 354, 56 (1991).

${ }^{2}$ L.F. Sun, S.S. Xie, W. Liu, W.Y. Zhou, Z.Q. Liu, D.S. Tang, G. Wang, and L.W. Qian, Nature (London) 403, 384 (2000).

${ }^{3}$ M.C. Gordillo, J. Boronat, and J. Casulleras, Phys. Rev. Lett. 85, 2348 (2000).

${ }^{4}$ M.C. Gordillo, J. Boronat, and J. Casulleras, Phys. Rev. B 61, R878 (2000).

${ }^{5}$ J. Boronat, M.C. Gordillo, and J. Casulleras, J. Low Temp. Phys. 126, 199 (2002).

${ }^{6}$ J. Tersoff and R.S. Ruoff, Phys. Rev. Lett. 73, 676 (1994).

${ }^{7}$ W. Teizer, R.B. Hallock, E. Dujardin, and T.W. Ebbesen, Phys. Rev. Lett. 82, 5305 (1999); 84, 1844 (2000).

${ }^{8}$ S. Talapatra, A.Z. Zambano, S.E. Weber, and A.D. Migone, Phys. Rev. Lett. 85, 138 (2000).

${ }^{9}$ E. Krotscheck, M.D. Miller, and J. Wojdylo, Phys. Rev. B 60, 13028 (1999).

${ }^{10}$ E. Krotscheck and M.D. Miller, Phys. Rev. B 60, 13038 (1999).

${ }^{11}$ M.W. Cole, V.H. Crespi, G. Stan, C. Ebner, J.M. Hartman, S. Moroni, and M. Boninsegni, Phys. Rev. Lett. 84, 3883 (2000).

${ }^{12}$ M. Boninsegni and S. Moroni, J. Low Temp. Phys. 118, 1 (2000).

${ }^{13}$ G. Stan et al., J. Low Temp. Phys. 113, 447 (1998).

${ }^{14}$ M.M. Calbi, M.W. Cole, S.M. Gatica, M.J. Bojan, and G. Stan, Rev. Mod. Phys. 73, 857 (2001).
${ }^{15}$ D.M. Ceperley, Rev. Mod. Phys. 67, 279 (1995).

${ }^{16}$ B.L. Hammond, W.A. Lester, Jr, and P.J. Reynolds, Monte Carlo Methods in Ab Initio Quantum Chemistry (World Scientific, Singapore, 1994).

${ }^{17}$ J. Boronat and J. Casulleras, Phys. Rev. B 49, 8920 (1994).

${ }^{18}$ R.A. Aziz and M.J. Slaman, Chem. Phys. 130, 187 (1989).

${ }^{19}$ L. Brualla, J. Boronat, and J. Casulleras, J. Low Temp. Phys. 126, 1547 (2002).

${ }^{20}$ M.C. Gordillo and D.M. Ceperley, J. Chem. Phys. 109, 2123 (1998).

${ }^{21}$ L. van Hove, Physica (Amsterdam) 16, 137 (1950).

${ }^{22}$ D. Ruelle, Commun. Math. Phys. 9, 267 (1968).

${ }^{23}$ F.J. Dyson, Commun. Math. Phys. 12, 91 (1969).

${ }^{24}$ E. Luijten and H. Messingfeld, Phys. Rev. Lett. 86, 5305 (2001).

${ }^{25}$ D.A. McQuarrie, Statistical Mechanics (University Science Books, Sausalito, CA, 2000).

${ }^{26}$ M.C. Gordillo, J. Boronat, and J. Casulleras, Phys. Rev. B 65, 014503 (2002).

${ }^{27}$ R. Radhakrishnan and K.E. Gubbins, Phys. Rev. Lett. 79, 2847 (1997).

${ }^{28}$ M.M. Calbi, S.M. Gatica, and M.W. Cole, Phys. Rev. B 67, 205417 (2003). 
${ }^{29}$ C. Carraro, Phys. Rev. B 61, R16 351 (2000).

${ }^{30}$ C. Carraro, Phys. Rev. Lett. 89, 115702 (2002).

${ }^{31}$ M.M. Calbi, F. Toigo, and M.W. Cole, Phys. Rev. Lett. 86, 5062 (2001).
${ }^{32}$ Lange's Handbook of Chemistry, edited by J. A. Dean, 14th ed. (McGraw-Hill, New York, 1992).

${ }^{33}$ R.E. Rapp, E.P. de Souza, and E. Lerner, Phys. Rev. B 24, 2196 (1981). 